\title{
The Effect of Regulatory Orientation and Decision Strategy on Brand Judgments
}

\author{
ECHO WEN WAN \\ JIEWEN HONG \\ BRIAN STERNTHAL*
}

\begin{abstract}
Four studies investigate how consumers' regulatory orientation and the decision strategies used to process message information affect their judgments. Evaluations of the chosen brand were more favorable when individuals with a prevention focus used decision strategies that enhanced the accuracy of a decision outcome than when they used strategies that facilitated progress toward a decision, whereas the opposite outcome occurred for those with a promotion focus. These findings emerged whether the decision strategies were prompted by instructions about how to make a decision or by the message presentation format, and they were mediated by a subjective experience of confidence. These observations suggest that judgments are influenced by the decision makers' feelings about how information is processed that are independent of the message content.
\end{abstract}

$I^{\mathrm{n}}$ nvestigations of consumer decision making suggest that choice is based on individuals' assessment of product attribute information that is guided by a decision strategy (Bettman, Luce, and Payne 1998; Payne, Bettman, and Johnson 1993). Depending on the context, the decision strategy might entail maximizing the accuracy of a judgment (e.g., equal weight strategy; Bettman et al. 1998) or facilitating rapid progress toward a decision (e.g., elimination-by-aspects [EBA] strategy, lexicographic; Bettman et al. 1998). In either case, judgments are content based: they rely on the concatenation of brand features as the basis for judgments. At the same time, there is emerging evidence that decisions can be based on metacognitions, which involve reflecting on the subjective experience of processing decision-related information and using this subjective experience to render a judgment (Aaker and Lee 2001; Higgins 2000; Lee and Labroo 2004; Tybout et al. 2005; Wänke, Bohner, and Jurkowitsch 1997). The purpose of the present research

*Echo Wen Wan is assistant professor of marketing, School of Business, University of Hong Kong, Pokfulam Road, Hong Kong (ewan@ business.hku .hk). Jiewen Hong is assistant professor of marketing, School of Business and Management, Hong Kong University of Science and Technology, Clearwater Bay, Kowloon, Hong Kong (mkjiewen@ust.hk). Brian Sternthal is Kraft Foods Chair Professor in Marketing, Kellogg School of Management, Northwestern University, 2001 Sheridan Road, Evanston, IL 60208-2001 (bst047@kellogg.northwestern.edu). The authors contributed equally. Correspondence: Echo Wen Wan. The authors thank Northwestern University and the University of Hong Kong for supporting this research.

John Deighton served as editor and Mary Frances Luce served as associate editor for this article.

Electronically published October 14, 2008 is to test the premise that the judgment of the chosen alternative is influenced by the correspondence between a decision maker's goal orientation and the specific strategy by which a decision is made. We refer to this prediction as the decision fit hypothesis.

To test this hypothesis, we vary two factors: the decision strategies used to make a judgment and the decision maker's goal orientation. The decision strategies are ones that prompt either the achievement of decision accuracy or the perception of rapid progress toward a decision. The goal orientation we investigate is regulatory focus (Higgins 1997), which pertains to two goals that are highly relevant in consumption contexts: prevention and promotion focus. We hypothesize that those with a prevention focus and the attendant desire for security experience fit when the decision strategy enhances the perception of decision accuracy, whereas for those with a promotion focus and the related desire for achievement, fit occurs when the decision strategy fosters the perception of rapid progress toward a decision. In turn, the presence of such correspondence induces a positive subjective experience that leads to a more favorable judgment of a chosen alternative than would occur in the absence of this correspondence.

\section{THE DECISION FIT HYPOTHESIS}

As a starting point in developing the rationale for the decision fit hypothesis, we describe the nature of regulatory focus orientations. According to regulatory focus theory (Higgins 1997), individuals with a prevention focus are concerned with safety, responsibilities, and obligations. They 
are sensitive to the presence and absence of negative outcomes and attempt to minimize errors of commission. In contrast, those with a promotion focus are concerned with growth, accomplishment, and aspirations. They are sensitive to the presence and absence of positive outcomes and attempt to minimize errors of omission (Crowe and Higgins 1997).

Our prediction of fit between those with a prevention focus and decision strategies that maximize the accuracy of the decision outcome is based on the observation that prevention-focused individuals are sensitive to the presence and absence of negative outcomes and attempt to minimize errors of commission (Crowe and Higgins 1997). This goal might be achieved by taking all available information into consideration in making a decision. Therefore, decision strategies that are perceived to maximize the accuracy of the decision outcome should fit with their regulatory orientation. However, the sensitivity of those with a promotion focus to positive outcomes and minimizing errors of omission suggests that fit would occur when decision strategies are perceived to facilitate rapid progress toward making a judgment. These decision strategies enhance the perception of those with a promotion focus that the opportunity for advancement has not been missed and that rapid progress is being made toward their goal.

Several studies are congenial with the decision fit hypothesis prediction that there is a correspondence between regulatory focus and the decision strategy related to accuracy versus progress. In one study, participants who were primed with either a prevention or a promotion focus were asked to connect the dots in four pictures as fast and as thoroughly as they could (Förster, Higgins, and Bianco 2003). They were told not to miss a dot and to get as much of the drawing done as possible within the allotted time. Prevention-focused participants exhibited greater accuracy in the drawing task than did those with a promotion focus, indicating the adoption of an accuracy strategy. In contrast, those with a promotion focus performed the task faster and were closer to completing the task, suggesting that they might have adopted a strategy that facilitates progress.

Additional support for the decision fit hypothesis was reported in a study in which participants were interrupted while in the process of describing an abstract figure (Liberman et al. 1999). After the interruption, participants were given a choice between resuming the task of describing the same figure as the one they had worked on before the interruption or switching to a task of describing a new figure. Participants with a prevention focus were more likely to continue to describe the same figure, whereas those with a promotion focus demonstrated a greater willingness to switch to a new description. Interpreted in terms of the decision fit hypothesis, prevention-focused participants continued to perform the task they had been working on because this strategy enhanced the chances of being accurate, whereas for promotion-focused participants switching to a new task created a greater sense of progress than adhering to the initial task.
The documentation of a correspondence between a prevention focus and decision strategies fostering accuracy and between a promotion focus and decision strategies facilitating rapid progress is of interest because it has implications for judgment. The decision fit hypothesis predicts that the correspondence between regulatory focus and a decision strategy is manifested by a more favorable evaluation of the chosen brand. Support for the prediction that fit between one's regulatory orientation and the means of goal pursuit leads to more favorable judgments has been found for a variety of responses such as message persuasiveness (Cesario, Grant, and Higgins 2004), liking of common objects (Higgins et al. 2003), and willingness to pay for a chosen object (Avnet and Higgins 2003). For example, Higgins et al. (2003) had participants choose between a coffee mug and an inferior pen using an eagerness or vigilance strategy that either fit or did not fit with their regulatory focus. Despite the fact that all participants chose the mug, their valuation of the mug was substantially greater when the strategy fit with their regulatory focus than when such fit was absent. These outcomes occurred despite the fact that all participants had the same information on which to base their judgments. These results are explained by the metacognitive notion that fit leads to more favorable evaluations by prompting a positive subjective experience that is manifested as processing fluency (Lee and Aaker 2004), feeling right (Higgins et al. 2003), and feeling confident (Cesario et al. 2004) and that is transferred to the chosen object. We adopt confidence as the label for the positive subjective experience in the present research.

In sum, the investigations reviewed offer credence for the decision fit hypothesis prediction that there is a fit between a prevention focus and decision strategies that maximize accuracy of a decision outcome and between a promotion focus and decision strategies that facilitate making rapid progress toward a decision. And this fit between individuals' regulatory focus and the strategy used to render a decision induces a positive subjective experience about the decision process that is manifested as more favorable evaluations of the chosen brand. The present research extends the literature on consumer decision making by going beyond contextual factors and exploring the interplay between individuals' selfregulatory orientation and decision strategies on brand evaluation. Support for the decision fit hypothesis predictions would also suggest when the impact of different decision strategies on the judgments can involve metacognitive processes in addition to the cognitive processes previously investigated.

\section{OVERVIEW OF THE CURRENT RESEARCH}

Four experiments were conducted to test the decision fit hypothesis. In all studies, participants were primed to adopt either a prevention or a promotion focus. Participants were then exposed to product information for two or more brands of products on a number of features. The information pre- 
sented was the same in all experimental conditions, but the decision strategy to be adopted in making a judgment was varied to fit with either a prevention or a promotion focus. To demonstrate the metacognitive nature of correspondence between regulatory focus and decision strategy, the stimuli were constructed so that there was a clearly dominant option that would be chosen regardless of the strategy used to make a decision. In studies 1 and 2, we tested the decision fit hypothesis by instructing participants to use a decision strategy that has been shown either to enhance decision accuracy (i.e., equal weight strategy) or to facilitate rapid progress toward making a decision (i.e., lexicographic strategy or EBA strategy; Bettman et al. 1998; Payne et al. 1993). In studies 3 and 4, we sought convergent evidence for the decision fit hypothesis by introducing presentation formats that might be used in everyday consumption settings to manipulate the decision strategies participants follow. Evidence regarding the process at work was sought by examining the role of the subjective experience of confidence in making a decision (studies 1 and 4).

\section{STUDY 1: FIT BETWEEN REGULATORY FOCUS AND DECISION STRATEGY}

The purpose of study 1 was to provide an initial test of the decision fit hypothesis. For this purpose, participants primed with either a promotion or a prevention focus were presented with product information for several brands of cell phones and were asked to make a choice among them and to evaluate the chosen alternative. Regulatory focus was primed by asking participants to think about avoiding negative outcomes in the prevention focus condition and to think about achieving positive outcomes in the promotion focus condition (Sengupta and Zhou 2007). Decision strategy was varied by presenting step-by-step instructions about how to process the product information. In the accuracy strategy condition, participants were given instructions that evoked an equal weight decision strategy. This strategy enabled them to take into consideration all pieces of information presented, thus maximizing the accuracy of the decision outcome (Bettman et al. 1998). In the progress strategy condition, participants were given instructions that evoked a lexicographic decision strategy (Bettman et al. 1998). This strategy has frequently been employed as an effort minimization strategy (Bettman et al. 1998), but it also can be characterized as facilitating rapid progress toward making a choice. Indeed, it has been found that both lexicographic and EBA strategies lead to faster response times than weighted-adding and equal weight strategies (Payne et al. 1993).

Thus, a 2 (regulatory focus: prevention vs. promotion) $\times$ 2 (decision strategy: accuracy [equal weight] vs. progress [lexicographic]) between-subject design was employed. The prediction is that those with a prevention focus would evaluate their chosen brand more favorably when using an equal weight decision strategy than when using a lexicographic strategy and that the reverse would occur for those with a promotion focus. These outcomes were anticipated because a correspondence between regulatory focus and decision strategy would prompt a subjective experience of confidence about the process by which a decision was made that would enhance the evaluation of the chosen brand. To test for this mediation, participants were asked to indicate their confidence about the process by which the decision was made (Cesario et al. 2004).

\section{Method}

Seventy-seven undergraduate students (39 female) from the University of Hong Kong participated in this study for 35 Hong Kong dollars (about 5 U.S. dollars). Regulatory focus was manipulated using an induction that was employed successfully with Asian participants (Sengupta and Zhou 2007). It entailed having participants respond to questions about an important course in which they were enrolled at the time. Those in the prevention focus condition were asked to write down a negative academic outcome that they wanted to avoid in the course and to describe the strategies they would use to avoid this outcome. Those in the promotion focus condition were asked to record a positive outcome they wished to achieve and to describe the strategies that they might use to achieve this outcome.

Upon completing the task, participants responded to two questions designed to check the adequacy of the regulatory focus manipulation: "To what extent did you focus on avoiding the negative outcome when writing down the academic strategies?" and "To what extent did you focus on achieving the positive outcome when writing down the academic strategies?" Responses were on 9-point scales $(1=$ not at all; 9 = very much).

Next, participants were asked to participate in an ostensibly unrelated study that involved choosing among five brands of cell phones (brands A, B, C, D, and E). All participants received information about four product features: battery talk time, battery standby time, warranty, and camera capability. The values on these features were such that brand B was superior to the other brands: it dominated each of the four alternative brands on at least one feature and was not dominated by any other brands on any feature (see appendix).

The decision strategy was manipulated by instructing participants at the outset of the choice task to use either an equal weight or a lexicographic strategy. The equal weight strategy followed the procedure that Avnet and Higgins (2003) adapted from commonly investigated decision strategies (Bettman et al. 1998). Participants in the equal weight strategy condition read instructions, as specified in the appendix, that required them to compare all brands of cell phone based on treating all attribute information equally. The instruction for the lexicographic strategy was adopted from Bettman et al. (1998). As specified in the appendix, the lexicographic strategy required participants to rank the importance of attributes and then compare all brands.

After examining the message information using the prescribed decision strategy, participants chose their preferred 
brand and then evaluated the chosen brand on four bipolar 7-point scales (bad/good, dislike/like a lot, unfavorable/favorable, unappealing/appealing), for which higher numbers indicated more favorable evaluations. This was followed by the administration of a series of 7-point scales that were designed to assess participants' mood when evaluating the cell phone (positive, content, negative [reverse coded], happy, calm, bad [reverse coded], and bored [reverse coded]; $1=$ not at all; $7=$ extremely). Finally, participants responded to the question, "How confident were you in making your choice of the preferred cell phone?" on a 7-point scale $(1=$ not at all confident; $7=$ very confident $)$.

A pretest was conducted to check the adequacy of the decision strategy manipulation. This pretest was conducted on 51 undergraduates from the same respondent pool as that recruited for the main study. They were given the same choice task as in the main study and were randomly assigned to either the equal weight or the lexicographic strategy condition. Participants were asked the extent to which they agreed that the decision strategy they used allowed them to make a quick decision on a 7-point scale $(1=$ strongly disagree; $7=$ strongly agree). The lexicographic strategy was perceived to allow for quicker decisions $(M=5.19$, $\mathrm{SD}=1.33)$ than the equal weight strategy $(M=4.42$, $\mathrm{SD}=1.21 ; F(1,49)=4.60, p<.05)$. Participants were also asked the extent to which they agreed that the decision strategy they used allowed them to make an accurate assessment of the alternative brands ( $1=$ strongly disagree; 7 = strongly agree). The equal weight strategy was perceived to result in a more accurate assessment of the alternative brands $(M=4.58, \mathrm{SD}=1.47)$ than the lexicographic strategy $(M=3.63, \mathrm{SD}=1.42 ; F(1,49)=5.55$, $p<.05)$. These results suggest that the manipulations of decision strategy were appropriate.

\section{Results and Discussion}

Manipulation Check. A one-way ANOVA indicated that participants primed with a prevention focus were more concerned about avoiding negative outcomes $(M=6.26$, $\mathrm{SD}=2.35)$ than achieving positive outcomes $(M=4.00$, $\mathrm{SD}=1.66 ; F(1,75)=22.44, p<.001)$, whereas those primed with a promotion focus were more concerned about achieving positive outcomes $(M=7.71, \mathrm{SD}=$ 1.11) than avoiding negative outcomes $(M=5.95, \mathrm{SD}=$ $1.73 ; F(1,75)=28.05, p<.001)$. These results suggest that our manipulation of regulatory focus was successful.

Evaluation. All participants chose the dominant brand (brand B) as their preferred brand. The four evaluation items loaded on a single factor and were averaged to form an evaluation score $(\alpha=.87)$. A $2 \times 2$ ANOVA indicated that neither the main effect of regulatory focus nor the main effect of decision strategy was significant $(F \mathrm{~s}<1)$. However, central to our hypothesis, the interaction between these factors was significant $(F(1,73)=15.59, p<.001$; see fig. 1$)$. As predicted, simple contrasts indicated that prevention-focused participants had more favorable evaluations of the
FIGURE 1

EVALUATION OF THE CHOSEN BRAND AS A FUNCTION OF REGULATORY FOCUS AND LEXICOGRAPHIC VERSUS EQUAL WEIGHT STRATEGY (STUDY 1)

Progress Strategy (Lexicographic)

$\square$ Accuracy Strategy (Equal Weight)

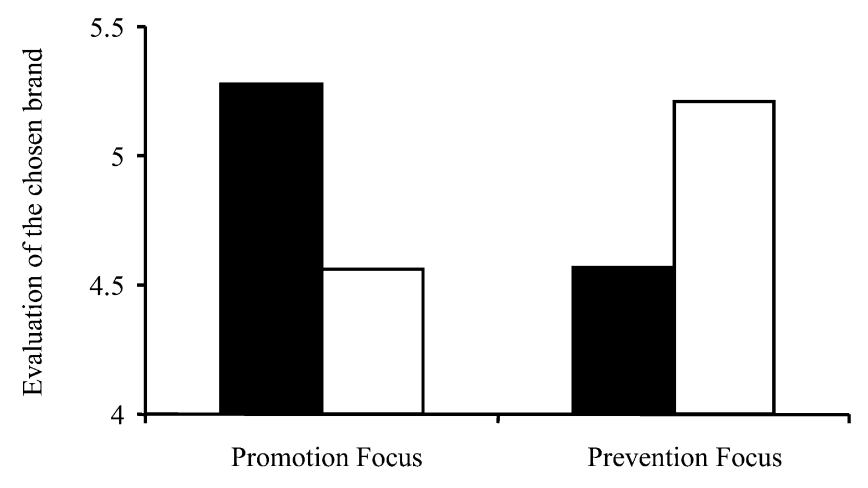

chosen brand when adopting the equal weight strategy $(M=5.21, \mathrm{SD}=.81)$ than the lexicographic strategy $(M=4.57, \mathrm{SD}=.80 ; F(1,73)=7.43, p<.01)$, whereas participants primed with a promotion focus exhibited more favorable evaluations when using the lexicographic strategy $(M=5.28, \mathrm{SD}=.70)$ than when using the equal weight strategy $(M=4.56, \mathrm{SD}=.63 ; F(1,73)=8.17, p<.01)$. In addition, when using the equal weight strategy, prevention-focused participants evaluated the chosen brand more favorably than the promotion-focused participants $\operatorname{did}(F(1,73)=7.03, p=.01)$, and the opposite outcome was found when the lexicographic strategy was used $(F(1,73)=8.60, p<.01)$.

Subjective Experience of Feeling Confident. To examine whether the subjective experience of feeling confident was implicated in explaining the effects of regulatory focus and decision strategy on evaluation, we first performed a $2 \times 2$ ANOVA on feelings of confidence. This analysis indicated that there were no significant main effects $\left(F_{\mathrm{s}}<1\right)$. However, the interaction between regulatory focus and decision strategy was significant $(F(1,73)=21.33, p<.001)$. Simple contrasts indicated that participants primed with a prevention focus felt more confident in making their choice when using the equal weight strategy $(M=5.52, \mathrm{SD}=$ 1.28) than when using the lexicographic strategy $(M=$ 4.06, $\mathrm{SD}=1.31 ; F(1,73)=16.61, p<.001)$, whereas those with a promotion focus reported being more confident when using the lexicographic strategy $(M=5.40, \mathrm{SD}=$ 1.04) than when using the equal weight strategy $(M=$ $4.44, \mathrm{SD}=.81 ; F(1,73)=6.30, p<.05)$. Moreover, when using the equal weight strategy, prevention-focused participants reported feeling more confident about making their choice than the promotion-focused participants did $(F(1,73)=16.61, p<.001)$, and the opposite was found 
when the decision strategy was lexicographic $(F(1,73)=$ $6.30, p<.05)$.

Next, we examined whether participants' subjective feeling of confidence mediated the effects of regulatory focus and decision strategy on evaluation. To examine the possibility that different mechanisms might underlie the subjective experience of prevention- and promotion-focused individuals, we conducted separate mediation analyses for those in each regulatory focus condition following the procedures developed by Baron and Kenny (1986). First, we tested whether feeling confident mediated the effect of decision strategy on the evaluations of those with a prevention focus. We regressed the evaluation score of the chosen brand on the dummy variable of decision strategy (equal weight $=0$; lexicographic $=1$ ). This analysis indicated that the equal weight strategy was associated with more favorable evaluations than the lexicographic strategy $(\beta=-.37, t(39)=-2.51, p<.05)$. Next, we regressed feelings of confidence on decision strategy, which indicated that the equal weight strategy induced a greater feeling of confidence than did the lexicographic strategy $(\beta=-.50$, $t(39)=-3.62, p=.001)$. This was followed by a regression of the evaluation score on feeling confident, which indicated that greater confidence was associated with more favorable evaluations $(\beta=.54, t(39)=3.96, p=.001)$. Finally, when evaluation was regressed on both decision strategy and confidence, the effect of confidence on evaluation remained significant $(\beta=.47, t(38)=2.97, p<$ $.01)$, whereas the effect of decision strategy was no longer significant $(\beta=-.14, t(38)=-.89, p=.38$; Sobel $z=$ $2.67, p<.01$; Sobel 1982). These results suggest that feeling confident mediated the effect of decision strategy on the evaluation of the chosen brand for participants primed with a prevention focus.

The same analysis was performed for those with a promotion focus. First, we observed that the lexicographic strategy was associated with more favorable evaluations than the equal weight strategy was $(\beta=.48, t(34)=3.20, p<.01)$. A second regression revealed that the lexicographic strategy prompted a greater feeling of confidence than did the equal weight strategy $(\beta=.46, t(34)=3.02, p<.01)$. Next, we observed that the feeling of confidence predicted evaluation $(\beta=.50, t(34)=3.40, p<.01)$. Finally, when both decision strategy and confidence were included in the model to predict evaluation, the effect of confidence on evaluation remained significant $(\beta=.37, t(33)=2.23, \quad p<.04)$, whereas the effect of decision strategy was significantly reduced $(\beta=.31, t(33)=1.98, p=.06$; Sobel $z=2.26$, $p<.05)$. These outcomes indicate that feeling confident partially mediated the effect of decision strategy on evaluation of the chosen brand for promotion-focused participants.

Mood. To examine whether mood accounted for the observed effects, we first performed a $2 \times 2$ ANOVA on the mood score $(\alpha=.75)$. This analysis yielded a marginally significant interaction effect $(F(1,73)=3.20, p<.08)$. No other effects were significant $(F \mathrm{~s}<1)$. Simple contrasts indicated that prevention-focused participants had a more positive mood when using the equal weight strategy $(M=4.91, \mathrm{SD}=.77)$ than when using the lexicographic strategy $(M=4.49, \mathrm{SD}=.88 ; F(1,73)=3.15, p=.08)$. Promotion-focused participants did not differ in their moods, whether using the equal weight strategy $(M=$ $4.62, \mathrm{SD}=.78)$ or the lexicographic strategy $(M=4.82$, $\mathrm{SD}=.58 ; F<1)$.

These results raise the possibility that the effect of decision strategy on the evaluations reported by those in the prevention focus condition might be attributed to differences in mood. To test this possibility, we examined whether mood mediated the effect of decision strategy in the prevention focus condition, following Baron and Kenny's (1986) procedure. First, a regression of the evaluation score on decision strategy (equal weight $=0$; lexicographic $=1$ ) indicated that the equal weight strategy was associated with more favorable evaluations than the lexicographic strategy $(\beta=-.37, t(39)=-2.51, p<$ $.05)$. Next, we regressed the mood score on decision strategy, which indicated no significant relationship between the two variables $(\beta=-.25, t(39)=-1.64, p=.11)$. These results indicate that the effect of decision strategy observed in the prevention focus condition is not accounted for by differences in mood.

We also performed a $2 \times 2$ ANCOVA on brand evaluation with mood as the covariate. Although mood was a significant covariate $(F(1,72)=31.52, p<.001)$, the same pattern of results was observed as in our earlier analysis in which mood was not included as a covariate. These outcomes suggest that the effects of regulatory focus and decision strategy on brand evaluation were independent of mood.

Study 1 provides evidence for the decision fit hypothesis. Participants primed with a prevention focus evaluated their choice more favorably when using an accuracy strategy (i.e., equal weight strategy) than a progress strategy (i.e., lexicographic strategy), whereas the opposite was found for those primed with a promotion focus. In addition, we documented that a subjective experience of feeling confident mediated the fit effects on evaluation: participants felt more confident about the decision process when using a decision strategy that fit with their regulatory focus, which in turn led to a more favorable evaluation of the chosen brand.

Although the data offer support for the decision fit hypothesis, the adequacy of the regulatory focus manipulation can be questioned. Having participants describe outcomes they wished to avoid and to achieve raises the possibility that approach/avoidance rather than regulatory focus was manipulated. To address this issue, we attempt to replicate our findings in study 2 using a different manipulation of regulatory focus. In addition, the robustness of our findings is assessed by recruiting American rather than Asian participants and by introducing an alternative means of varying the progress decision strategy. 


\section{STUDY 2: THE ROBUSTNESS OF EFFECTS}

Study 2 was designed to assess the robustness of the decision fit hypothesis using procedures similar to those employed in study 1. Participants were initially primed with either a promotion or a prevention focus using a word completion task. This was followed by the presentation of the same product information for several brands of cell phones as was used in the previous study. Decision strategy was again varied by presenting step-by-step instructions about how to process the product information. In the accuracy strategy condition, participants were given the same instructions as used in study 1 to stimulate the use of an equal weight decision strategy, whereas in the progress strategy condition the instructions prompted an EBA strategy rather than the lexicographic strategy used previously. Thus, a 2 (regulatory focus: prevention vs. promotion) $\times 2$ (decision strategy: accuracy [equal weight] vs. progress [EBA]) between-subject design was employed. Participants' evaluation of the chosen brand served as the key dependent variable. The prediction is that those with a prevention focus would evaluate their chosen brand more favorably when using an equal weight decision strategy than when using an EBA strategy, and the reverse would occur for those with a promotion focus.

\section{Method}

Sixty-four undergraduate students (32 female) from Northwestern University participated in this study for course credit. Regulatory focus was manipulated using a word fragment completion task (Gilbert and Hixon 1991). This task involves a mind-set manipulation that temporarily activates a promotion or prevention focus. In the prevention focus condition, four words related to vigilance served as stimuli (secure, calm, vigilant, safe), whereas in the promotion focus condition, the four words were associated with eagerness (eager, active, growth, accomplish). For each word, one or two letters were missing. For example, for eager the $a$ and $e$ were missing (i.e., e_g_r), and for secure the two $e$ 's were missing. The participants' task was to fill in the missing letters for each of the four words presented.

The regulatory focus induction was followed by the same product description as was used in study 1. Participants then were administered the decision strategy manipulations employed by Avnet and Higgins (2003). The accuracy decision strategy was manipulated using the same equal weight instructions as those previously used. For the progress decision strategy, the EBA instruction read: "Please examine the information about these brands of cell phone using the following strategy. Look at the first attribute, Battery Talk Time, brand by brand. Exclude the brand that has the worst value on this attribute. Now you are left with four brands. Go to the second attribute, Battery Standby Time, and again look at it for all the remaining brands. Exclude the brand that has the worst value on this attribute. Follow this procedure until you are left with only one brand. Mark it as your chosen brand."
After participants chose the brand they preferred, they were asked to evaluate the chosen brand on a series of 7point scales. The evaluation items included the four administered in study 1 as well as three new items. Of these, three were bipolar items $(1=$ bad, ordinary, dislike; $7=$ extremely good, state of the art, like a lot), and four were unipolar items (favorable, revolutionary, superior, unappealing [reverse coded]; $1=$ not at all; $7=$ extremely). Finally, participants reported their mood using the same scales as in study 1 .

A pretest conducted on 62 undergraduates from the same respondent pool as the one used in the main study provided evidence for the adequacy of the decision strategy manipulation. Participants were given the same choice task as in the main study and were randomly assigned to either the equal weight or the EBA strategy condition. Participants were asked to indicate on a 7-point scale $(1=$ strongly disagree; $7=$ strongly agree) the extent to which they agreed that the decision strategy they used allowed them to make a quick decision. The EBA decision strategy was perceived to allow for a quicker decision $(M=6.39, \mathrm{SD}=$ 1.17) than the equal weight strategy allowed $(M=5.35$, $\mathrm{SD}=1.43 ; F(1,60)=9.67, p<.01)$. Participants were also asked the extent to which they agreed that the decision strategy they used allowed them to make an accurate assessment of the alternative brands ( $1=$ strongly disagree; 7 = strongly agree). The equal weight strategy was perceived to enable a more accurate assessment of the alternative brands $(M=5.61, \mathrm{SD}=1.50)$ than the EBA strategy $\operatorname{did}(M=4.65, \mathrm{SD}=2.17 ; F(1,60)=4.18, p<.05)$. These results suggest that our manipulations of decision strategy were adequate.

\section{Results and Discussion}

Evaluation. All participants chose the dominant brand (brand B) as their preferred brand. The seven evaluation items pertaining to brand $\mathrm{B}$ loaded on a single factor and were averaged to form an evaluation score $(\alpha=.70)$. A $2 \times 2$ ANOVA on the evaluation score indicated that the main effects of regulatory focus and decision strategy were not significant $\left(F_{\mathrm{S}}<1\right)$. However, the interaction between these factors was significant $(F(1,60)=14.93, p<.001)$. As figure 2 shows, participants primed with a prevention focus evaluated the chosen brand more favorably when using an equal weight strategy $(M=5.26, \mathrm{SD}=.78)$ than when using an EBA strategy $(M=4.51, \mathrm{SD}=.49 ; F(1,60)=$ $9.64, p<.01)$. In contrast, those primed with a promotion focus had more favorable evaluations when using an EBA strategy $(M=5.03, \mathrm{SD}=.55)$ than when using an equal weight strategy $(M=4.46, \mathrm{SD}=.83 ; F(1,60)=5.55$, $p<.05)$. Moreover, prevention-focused participants were more favorable toward the chosen brand than promotionfocused participants were when using an equal weight strategy $(F(1,60)=10.24, p<.01)$, whereas promotion-focused participants evaluated the chosen brand more favorably than prevention-focused participants did when following an EBA 


\section{FIGURE 2}

EVALUATION OF THE CHOSEN BRAND AS A FUNCTION OF REGULATORY FOCUS AND ELIMINATION-BY-ASPECTS VERSUS EQUAL WEIGHT STRATEGY (STUDY 2)

Progress Strategy (Elimination-by-Aspects) $\square$ Accuracy Strategy (Equal Weight)

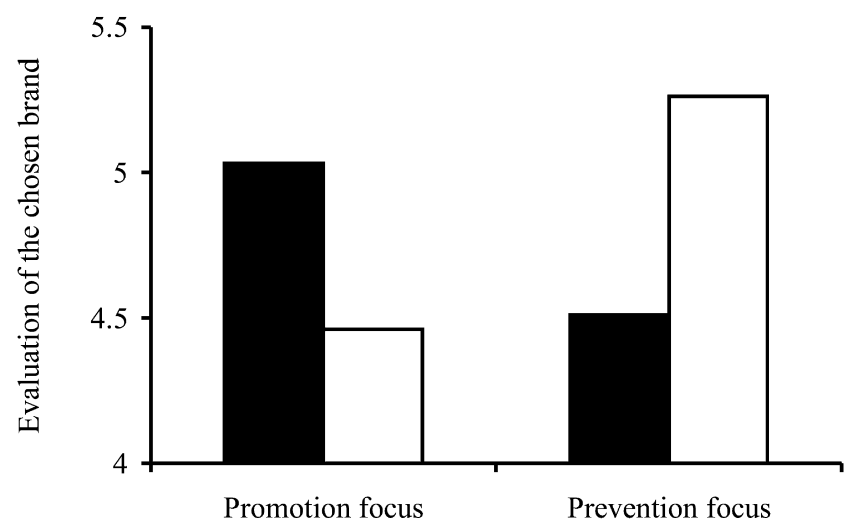

strategy $(F(1,60)=5.00, p<.05)$. These results provide support for the decision fit hypothesis.

Mood. A mood score was obtained by averaging responses to the seven mood items that loaded on a single factor $(\alpha=.71)$. To assess the role of mood in influencing brand evaluations, a $2 \times 2$ ANOVA was performed on the mood score. This analysis yielded no significant effects $(F \mathrm{~s}<1)$. We also performed a $2 \times 2$ ANCOVA on brand evaluation using mood as a covariate. The results showed that although mood was a significant covariate $(F(1,59)=$ $20.26, p<.001)$, the same pattern of results emerged as in our earlier analysis in which mood was not included as a covariate. Thus, consistent with prior research, these results suggest that the effect on evaluation was independent of mood (Cesario et al. 2004).

As predicted by the decision fit hypothesis, the results from study 1 showed that despite the choice of the same (dominant) brand by all participants, evaluation of the chosen brand was more favorable when prevention-focused participants employed an accuracy decision strategy (i.e., equal weight strategy) than when using a progress strategy (i.e., EBA strategy); the opposite result was found for those primed with a promotion focus. Moreover, the effects on evaluations were not accounted for by differences in mood.

Studies 1 and 2 tested the decision fit hypothesis by instructing participants to follow procedures that evoked accuracy versus progress decision strategies. However, in everyday consumption situations, it is typically difficult to instruct consumers regarding the procedures they should follow in making their decisions. Rather, consumer decision strategies are often influenced by how information is presented. In studies 3 and 4, we attempt to enhance the applicability of our findings by manipulating message presen- tation format as a means of varying accuracy and progress decision strategies.

\section{STUDY 3: USING PRESENTATION FORMAT TO VARY DECISION STRATEGY}

We introduced two changes in procedure to test the decision fit hypothesis in study 3. First, we used a different manipulation of regulatory focus than those employed in the previous studies to assess the robustness of our findings. We also introduced a new manipulation of decision strategies. Instead of using instructions to prompt participants to employ either an accuracy or a progress decision strategy, we employed a variation in the message presentation format to induce different decision strategies. This entailed either a simultaneous presentation of product information about two brands in which the information about their performance on all features was presented on a single page or a sequential presentation in which the product features were presented sequentially on several pages.

Although there is no precedence for the use of simultaneous and sequential presentations to operationalize accuracy and progress decision strategies, there are theoretical and practical grounds for selecting these manipulations. We reasoned that presenting all of the information about the alternatives simultaneously on one page would facilitate the detailed comparison of all pieces of information, which in turn would enhance the perception that the decision would be accurate. In contrast, accumulating information sequentially on different pages highlights the feeling that new information is gained on each page, which would promote the perception of achieving progress toward a decision. Consistent with this analysis is the finding that when the decision makers' goal was accurate assessment, they exhibited a tendency to restructure sequentially presented information so that it was displayed simultaneously (Coupey 1994). Also consistent with our rationale for the manipulation is the observation that a sequential presentation of information stimulates the use of a lexicographic decision strategy (Broder and Schiffer 2003), which as we noted in study 1 enhances the perception of rapid progress. From a practical perspective, simultaneous and sequential presentation formats are often used in advertising. For example, Internet shopping Web sites frequently offer the option of presenting the product features of alternative options on one page or showing the feature information that consumers are interested in step by step. In television advertising, the superiority of one brand over another is sometimes presented all at once (often using a chart) and at other times presented by a spokesperson comparing alternatives one feature at a time.

Thus, a 2 (regulatory focus: prevention vs. promotion) $\times$ 2 (presentation format: simultaneous [accuracy strategy] vs. sequential [progress strategy]) between-subject design was employed. The prediction is that individuals with a prevention focus would evaluate the chosen brand more favorably when product information was presented in a simultaneous 
rather than a sequential format, and the opposite outcome would occur for those with a promotion focus.

\section{Method}

Eighty-four undergraduate students (42 female) from Northwestern University were paid $\$ 10$ for their participation in this study. Participants were informed that they would be completing a number of separate studies. The first task was described as an emotional empathy study, which had been used successfully to vary regulatory focus in previous research (Friedman and Förster 2001). Participants were asked to solve a maze task in which a mouse positioned in the middle of a maze was seeking a path to leave the maze. In the promotion focus condition, the motivation to leave the maze was to get to the cheese, whereas in the prevention focus condition the motivation was to escape from an owl that was lurking overhead. All participants completed this task successfully. They were then asked to describe their goals when taking the perspective of the mouse. This served as the check for the regulatory focus manipulation.

Immediately after the regulatory focus manipulation, participants were asked to examine information about two brands of computer, A and B. They were given product information about the two brands presented in a side-byside comparison of the features. The message included information about nine features, such that brand B dominated brand $\mathrm{A}$ on two features (monitor size and hard drive capacity), brand A dominated brand $\mathrm{B}$ on one feature (warranty), and the two brands were at parity on the remaining six features (battery life, DVD ROM, memory capacity, encyclopedia, DVD burner, and 24-hour hotline). Thus, overall, brand B was the dominant brand. Decision strategy was varied by the format used to present the information. In the simultaneous presentation condition, a comparison of the brands on all nine features was presented on a single page. The features on which brand $\mathrm{B}$ dominated were listed first and sixth, and the one feature on which brand A dominated was listed fourth. In the sequential presentation condition, the product features were presented in the same order, but the information was presented on three pages. Information about the first two features (monitor size and battery life) was presented on the first page. The next page included the description of these features as well as three additional features (DVD ROM, warranty, and memory capacity). The third page included information about all of the features and thus was identical to the information presented in the simultaneous presentation condition. After reading the product information, participants indicated their preferred brand, evaluated their chosen brand, and reported their mood on the same items as those used in study 2.

A pretest was conducted to examine the adequacy of simultaneous and sequential presentation formats as a manipulation of decision strategies. Thirty-five students recruited from the same respondent pool as the one used in the main study were assigned to either the simultaneous or the sequential presentation condition. They were asked to indicate on a 7 -point scale $(1=$ strongly disagree; $7=$ strongly agree) the extent to which they agreed that the way the information was presented made them feel that they were rapidly progressing toward choosing the best option. The sequential presentation was perceived to allow for a quicker decision $(M=5.79, \mathrm{SD}=.85)$ than the simultaneous presentation allowed $(M=4.76, \mathrm{SD}=1.50 ; F(1,34)=6.14$, $p<.05)$. Participants were also asked the extent to which the way the information was presented allowed them to make an accurate assessment of the alternative brands. The simultaneous presentation $(M=5.06, \mathrm{SD}=1.43)$ was perceived to enable a more accurate assessment of the alternative brands than the sequential presentation did $(M=4.11, \mathrm{SD}=$ $1.28 ; F(1,34)=4.42, p<.05)$. These results suggest that the presentation formats manipulated accuracy and progress decision strategies.

\section{Results and Discussion}

Manipulation Checks. To assess the adequacy of the regulatory focus manipulation, participants' responses pertaining to their goal in taking the perspective of a mouse were coded by two judges. The total number of responses that implied a promotion or a prevention focus was recorded. For example, "my goal was to eat the cheese to satisfy my hunger" was coded as indicating a promotion focus, and "my goal was to get out of the maze safely" was coded as representing a prevention focus. The two judges agreed in their classification $97 \%$ of the time. Disagreement was resolved by a discussion between the judges. Two separate one-way ANOVAs examined the effect of the regulatory focus manipulation on the number of participants' responses that implied a promotion or a prevention focus. The results indicated that those primed with a promotion focus listed more promotion-oriented $(M=.88, \mathrm{SD}=.34)$ than preventionoriented responses $(M=.24, \mathrm{SD}=.43 ; F(1,76)=53.64$, $p<.001)$. In contrast, those primed with a prevention focus reported more prevention-oriented $(M=.58, \mathrm{SD}=.50)$ than promotion-oriented responses $(M=.05, \mathrm{SD}=.22$; $F(1,76)=37.12, p<.001)$. These results suggest that the manipulation of regulatory focus was successful.

Evaluation. All participants chose the dominant brand (brand B). The seven evaluation items loaded on a single factor and were averaged to form an evaluation score $(\alpha=.85)$. A $2 \times 2$ ANOVA indicated that the main effects of regulatory focus and presentation format were not significant $(F \mathrm{~s}<1)$. However, the interaction between these factors was significant $(F(1,80)=10.71, p<.01$; see fig. 3$)$. As predicted, for participants with a prevention focus, evaluations were more favorable when information was presented simultaneously $(M=5.22, \mathrm{SD}=.18)$ than when it was presented sequentially $(M=4.57, \quad \mathrm{SD}=.18 ; \quad F(1,80)=$ $6.58, p<.05)$. In contrast, those primed to adopt a promotion focus had more favorable evaluations when the product information was presented sequentially $(M=5.18, \mathrm{SD}=$ .17) than when it was presented simultaneously $(M=$ $4.66, \mathrm{SD}=.18 ; F(1,80)=4.24, p<.05)$. Moreover, when 


\section{FIGURE 3}

EVALUATION OF THE CHOSEN BRAND AS A FUNCTION OF REGULATORY FOCUS AND SEQUENTIAL VERSUS SIMULTANEOUS PRESENTATION (STUDY 3)

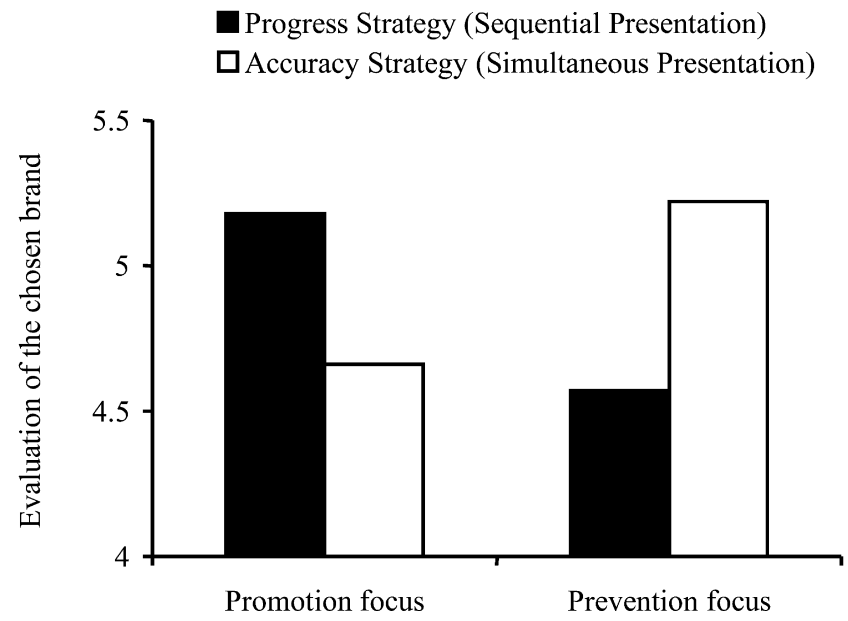

the information presentation was simultaneous, preventionfocused participants had more favorable evaluations than those with a promotion focus $(F(1,80)=4.62, p<.05)$. In contrast, when the presentation format was sequential, promotion-focused participants were more favorable toward their chosen brand than prevention-focused participants were $(F(1,80)=6.19, p<.05)$.

Mood. We first performed a $2 \times 2$ ANOVA on the mood score $(\alpha=.74)$. This analysis yielded no significant effects ( $p$ s $>.14)$. Moreover, a $2 \times 2$ ANCOVA on evaluation with mood as the covariate yielded the same pattern of results as we observed in our earlier analysis in which mood was not included, even though mood was a significant covariate $(F(1,79)=31.39, p<.001)$. These results again suggest that the effect of fit on evaluation was independent of mood.

We also assessed the possibility that prevention-focused participants were less favorable toward the chosen brand when the presentation was sequential rather than simultaneous because they found the repetitive presentation of some of the features to be unpleasant. If this were the case, there should be a difference in mood prompted by the simultaneous and sequential presentation formats among those with a prevention focus. To examine this possibility, we regressed mood on presentation format (sequential = 0 ; simultaneous $=1$ ) for prevention-focused participants. This analysis indicated that mood was not significantly affected by the presentation format $(\beta=-.07, t<1)$, suggesting that mood did not account for the effect of presentation format on the evaluations of prevention-focused participants.

The results of study 3 have both theoretical and practical implications. From a theoretical perspective, the findings provide additional evidence that fit between regulatory focus and decision strategies related to accuracy and progress leads to more favorable evaluations of the chosen brand. Evaluations were more favorable for those primed with a prevention focus when the presentation of the features was simultaneous than when it was sequential, whereas the opposite pattern was observed for those with a promotion focus. From a practical perspective, the findings demonstrate that decision strategies can be created in everyday settings to fit with different regulatory orientations so that they lead to more favorable evaluations.

\section{STUDY 4: THE NATURE OF THE FIT EFFECT}

Study 4 was designed to achieve three objectives. One was to examine whether the effects of fit between regulatory focus and presentation format demonstrated in study 3 were mediated by a positive subjective experience in processing the message. As in study 1, we measured participants' feeling of confidence in making their choice as an indicator of their subjective experience. A second objective was to assess the effects of fit on both the chosen and the nonchosen brand. This would enable us to determine whether the effects of fit between regulatory focus and decision strategy were specific to the brand selected or whether fit created a general positive subjective experience that affected both the chosen and the nonchosen alternatives. To control for the potential effect of the order in which the evaluations of the two brands were administered, we counterbalanced the order of these evaluations. Finally, to rule out the possibility that the fit effects observed in study 3 might be attributed to the fact that the dominant features were placed at the outset of the message, which might bias the evaluation of subsequent features (Carlson, Meloy, and Russo 2006), we listed the features on which the superior brand dominated the alternative at the end of the feature list rather than earlier as we had in study 3. Thus, a 2 (regulatory focus: prevention vs. promotion) $\times 2$ (presentation format: simultaneous [accuracy strategy] vs. sequential [progress strategy]) $\times 2$ (order of brand evaluation: brand A first vs. brand B first) betweensubject design was employed.

\section{Method}

One hundred and eight undergraduate students (55 female) from Northwestern University were each paid $\$ 10$ for their participation. First, participants were administered the same word fragment completion task as was used in study 2 to manipulate regulatory focus. This was followed by an ostensibly unrelated study in which participants were asked to choose from two brands of cell phone, A and B. They were presented with information comparing the two brands on seven features (in the order of presentation): battery standby time, weight, camera capability, warranty, voice recorder function, battery talk time, and phone book capacity. The performance of the two brands was equivalent on the first five features, and brand $\mathrm{A}$ was superior to brand $\mathrm{B}$ on the last two features. In the simultaneous presentation con- 
dition, all seven features were presented on a single page. In the sequential presentation condition, the features were presented sequentially on three pages. The first two features were presented on the first page, the first two plus the following two features were presented on the second page, and the first four plus the remaining three features were presented on the third page. This last page thus exhibited the same information as was presented in the simultaneous condition.

After reading the product information, participants indicated their preferred brand and evaluated both the chosen and the alternative brand on the same four items as were used in study 1. About half the participants evaluated brand A first and then brand B. The remaining participants evaluated the brands in the reverse order. Then all participants completed the seven mood items used in our previous studies. Finally, they reported their subjective experience by indicating the extent to which they felt confident in making their choice on a 7 -point scale $(1=$ not at all confident; $7=$ very confident $)$.

\section{Results and Discussion}

Evaluation of the Chosen Brand. All participants chose the dominant brand (brand A). Responses to the four items on which participants evaluated the chosen brand were averaged to form an evaluation score $(\alpha=.85)$. A $2 \times$ $2 \times 2$ ANOVA indicated that the three-way interaction was not significant $(F<1)$, suggesting that the order of brand evaluation did not affect participants' evaluation of the chosen brand. However, the predicted interaction between regulatory focus and presentation format was significant $(F(1,100)=9.58, p<.01)$. As shown in figure 4 , prevention-focused participants had more favorable evaluations of the chosen brand when the presentation was simultaneous $(M=5.60, \mathrm{SD}=.14)$ than when it was sequential $(M=5.14, \mathrm{SD}=.14 ; F(1,100)=5.35, p<.05)$, whereas promotion-focused participants evaluated their chosen brand more favorably when the presentation was sequential $(M=5.62, \mathrm{SD}=.15)$ than when it was simultaneous $(M=5.18, \mathrm{SD}=.14 ; F(1,100)=4.30, p<.05)$. Moreover, in the simultaneous presentation condition, those with a prevention focus had more favorable evaluations than those with a promotion focus $(F(1,100)=4.52, p<.05)$, and the reverse occurred in the sequential presentation condition $(F(1,100)=5.06, p<.05)$.

Evaluation of the Nonchosen Brand. Responses to the four items on which participants evaluated the nonchosen brand (brand $\mathrm{B}$ ) were averaged to form an evaluation score $(\alpha=.84)$. A $2 \times 2 \times 2$ ANOVA indicated a significant main effect of brand order: participants evaluated brand $B$ more favorably when it was evaluated prior to brand A $(M=4.77, \mathrm{SD}=.96)$ than when it was evaluated after brand A $(M=4.22, \mathrm{SD}=.94 ; F(1,98)=8.85, p<.01)$. No other effects were significant $(p s>.16)$. Apparently, evaluating brand $\mathrm{B}$ after brand $\mathrm{A}$ highlighted the fact that brand $\mathrm{B}$ was inferior to brand $\mathrm{A}$.
FIGURE 4

EVALUATION OF THE CHOSEN BRAND AS A FUNCTION OF REGULATORY FOCUS AND SEQUENTIAL VERSUS SIMULTANEOUS PRESENTATION (STUDY 4)

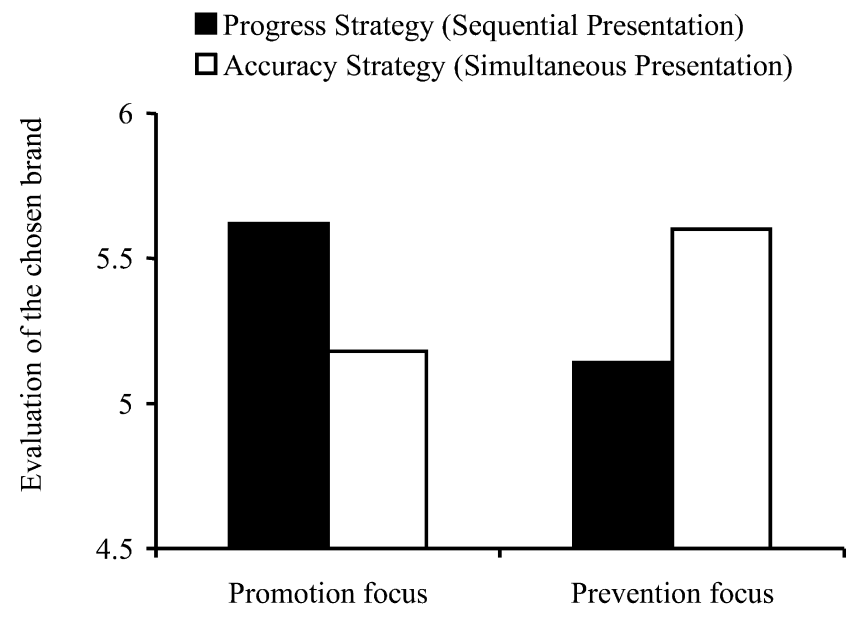

Subjective Experience of Feeling Confident. Given that there was no order effect in the evaluation of the chosen brand, we performed a 2 (regulatory focus) $\times 2$ (presentation format) ANOVA on participants' feeling of confidence in making their choice. This analysis indicated that neither the main effect of regulatory focus nor that of presentation format was significant $(p s>.21)$. However, the interaction between these factors was significant $(F(1,104)=5.26$, $p<.05)$. Prevention-focused participants felt more confident in their choice when the presentation was simultaneous $(M=5.77, \mathrm{SD}=1.50)$ than when it was sequential $(M=4.85, \mathrm{SD}=1.81 ; F(1,104)=5.37, p<.05)$. However, in contrast to the findings in study 2 , those with a promotion focus did not report greater confidence when the presentation was sequential $(M=5.87, \mathrm{SD}=.96)$ than when it was simultaneous $(M=5.46, \mathrm{SD}=1.47 ; F<1)$.

Given that the feeling of confidence did not vary between the simultaneous and the sequential presentations for promotion-focused participants, we only examined whether feeling confident mediated the effect of presentation format on the evaluation of the chosen brand in the prevention focus condition. First, we regressed the evaluation score on presentation format (sequential presentation $=0$; simultaneous presentation $=1$ ). This analysis indicated that the simultaneous presentation led to more favorable evaluations than the sequential presentation $\operatorname{did}(\beta=.31, t(55)=2.39$, $p<.05)$. Next, we regressed feelings of confidence on presentation format, which indicated that the simultaneous presentation was associated with greater confidence $(\beta=.27$, $t(55)=2.08, p<.05)$. This was followed by a regression of evaluation on feeling confident, which indicated that greater feelings of confidence led to more favorable evaluations $(\beta=.37, t(55)=2.96, p<.01)$. Finally, a regression analysis using both presentation format and feeling confident to predict evaluation showed that the effect of 
feeling confident on evaluation remained significant $(\beta=$ $.31, t(54)=2.43, p<.05)$, while the effect of presentation format became marginally significant $(\beta=.22, t(54)=$ $1.75, p=.09$; Sobel $z=1.72, p=.08$ ). These outcomes suggest that for prevention-focused participants the subjective experience of feeling confident partially mediated the effect of presentation format on evaluations.

Mood. A $2 \times 2$ ANOVA on the mood index $(\alpha=$ .79) yielded no significant effects $(p s>.14)$. Moreover, a $2 \times 2 \times 2$ ANCOVA on the evaluation of the chosen brand with mood as the covariate yielded the same pattern of results as found in our earlier analysis in which mood was not included as a covariate and indicated that mood was not a significant covariate $(F<1)$. These outcomes suggest that the fit effect on evaluation was not attributable to mood.

To assess the possibility that the sequential presentation stimulated a negative mood and the resulting unfavorable evaluations of the chosen brand for those with a prevention focus, we examined whether presentation format predicted mood in the prevention focus condition. A regression on mood with presentation format as the predictor (sequential presentation $=0$; simultaneous presentation $=1$ ) indicated that prevention-focused participants did not differ in their mood whether the presentation format was sequential or simultaneous $(\beta=.20, t(55)=1.50, p>.10)$. This result suggests that the effect of presentation format on prevention-focused participants' brand evaluations could not be explained by differences in mood.

The results of study 4 replicated the effects of fit reported in our earlier studies. Participants with a prevention focus evaluated their choice more favorably when information was presented simultaneously than when the same information was presented sequentially, and the reverse occurred for promotion-focused participants. Moreover, these outcomes occurred when the features on which the chosen brand dominated were presented last. In addition, we documented that for those with a prevention focus the effect of fit on evaluations was mediated by the subjective experience of feeling confident in making their choice.

It is noteworthy that confidence did not mediate the effect of presentation formation for those with a promotion focus. This finding differs from that found in study 1 in which confidence mediated the effects of fit on evaluations for both promotion- and prevention-focused participants. A contributing factor to this disparity might be related to the participants in the two studies. Our participants in study 1 were Asians, whereas the participants in the present study were Americans. Prior research suggests that in contrast to Asians, Americans tend to have greater confidence in general (e.g., Heine et al. 1999), and thus they might feel uniformly confident irrespective of the fit with the presentation format. The supposition is consistent with the high confidence scores exhibited by promotion-focused participants in response to both presentation formats. These observations suggest that the null effect of presentation format on feelings of confidence for promotion-focused participants might be attributed to a ceiling effect.
Finally, we observed that the evaluation of the nonchosen brand was not affected by fit. This outcome might seem to be at odds with demonstrations that fit effects can be transferred to the evaluations of unrelated objects (e.g., Higgins 2000; Higgins et al. 2003). For example, creating fit between promotion and eagerness activities and prevention and vigilance activities affected the subsequent evaluation of a dog (Higgins et al. 2003). In contrast, we found that fit affected the evaluations of the dominant brand but not the alternative brand. This outcome may have occurred because participants recognized that the dominated brand was inferior in comparison to the dominant brand, which made them attentive to the fact that the subjective experience of fit did not apply to the dominated alternative.

\section{GENERAL DISCUSSION}

The present research provides support for the decision fit hypothesis. In four studies, we observed that those primed to adopt a prevention focus evaluated their choice more favorably when the decision was made using a strategy that enhanced the perception of accuracy than a sense of rapid progress, and those primed to adopt a promotion focus exhibited the opposite pattern. These outcomes occurred whether the decision strategies related to accuracy and progress were manipulated by the instructions about how to process the message or by the message presentation format. Also consistent with the decision fit hypothesis is the finding that a feeling of confidence mediated the effect of fit on evaluations, although this did not occur reliably for those with a promotion focus.

The present research contributes to the literature on consumer decision making. Extant research on decision making has primarily focused on how the context in which the decision is made influences decision strategies, such as task importance (Bettman et al. 1998) and time pressure (Payne, Bettman, and Johnson 1988). We extend this analysis by demonstrating that decision makers' self-regulatory orientation affects the impact of strategies that maximize accuracy (e.g., equal weight strategy) and facilitate rapid progress toward making a decision (e.g., lexicographic and EBA) on evaluation of the decision outcome. In doing so, we document that brand evaluations depend not only on how stimulus information is concatenated under different decision strategies (Bettman et al. 1998; Payne et al. 1988) but also on the decision makers' subjective experience of confidence that results from a reflection on the decision process.

The current research also contributes to the regulatory fit literature. Whereas prior research has primarily examined eagerness and vigilance strategies (see Avnet and Higgins [2003] for an exception), the current research advances the analysis of regulatory focus by linking it to two important types of decision strategies (accuracy strategy and progress strategy). In addition, the present research extends the work of Avnet and Higgins (2003), which demonstrated a fit between regulatory mode (i.e., locomotion vs. assessment) and decision strategies (full evaluation vs. progressive elimination) by showing that ( $a$ ) fit can also occur between reg- 
ulatory focus and these decision strategies, $(b)$ fit can occur between regulatory focus and alternative presentation formats that foster accuracy and progress, $(c)$ the subjective experience of confidence mediates the fit effects, and $(d)$ the effects of fit do not extend to the nonchosen brand.

More generally, our findings regarding the effects of fit on evaluation add to the growing body of research that converges on the view that judgments are influenced by two distinct processes (Carver and Scheier 1998; Kahneman and Frederick 2002; Schwarz 2004; Sloman 1996). One is cognitive. Here, memory is used to select the information that serves as the basis for a judgment. Prior research examining the impact of decision strategies is represented in this category. The other process is metacognitive. It involves an assessment of the judgment process in which memory is reflective about how the judgment is being made. Our findings offer evidence that evaluations can be based on metacognitive processes. Viewed from this perspective, a fit between regulatory focus and a decision strategy activates a positive subjective experience that is transferred to the evaluation of the dominant choice alternative. The fact that in all of our studies participants were exposed to the same message content is consistent with the notion that the evaluations were based on metacognitions. Support for the operation of metacognitive processes is also documented by the finding that a subjective experience of confidence about how the decision was made mediated the effects of fit on evaluations. Our findings also suggest that the metacognitive process is activated even in situations in which the message content offers an unambiguous basis for judgment. Indeed, in studies 1, 2, and 4, it was evident that one brand had asymmetric dominance over the alternative(s). Yet, we still observed the effects of fit on evaluations of the chosen brand. This observation suggests that ambiguity might not be a necessary factor in determining whether metacognitive processes would be activated.

The findings from the current research also have applications in everyday consumer settings. For example, consider the toothpaste category in which a brand such as Colgate Total is positioned as preventing tooth decay. This position implies that those with a prevention focus would be targeted. Our research suggests that using presentation formats that facilitate complete comparison of alternatives, such as a simultaneous presentation, would increase the impact of messages for this brand. In contrast, for brands positioned as whitening teeth such as Rembrandt, the likely target is individuals with a promotion focus, whose goal is to have a bright smile. Here, our research implies that a message prompting a perception of progress toward a decision would increase the persuasiveness of appeals for such brands.

A recent launch of a new toothpaste brand provides additional credence for the decision fit hypothesis. The brand targets those who are determined to avoid problems in dental hygiene. These people not only brush their teeth several times a day but also floss regularly and use mouthwash as well as various implements to avoid cavities and gum dis- ease. Thus, the target segment is likely to adopt a prevention focus in choosing a toothpaste. Two spots were pretested to stimulate brand trial. One involves the simultaneous presentation of the new brand's features. Here, the seven areas dental hygiene dentists are most concerned about are listed (e.g., plaque, bad breath, tartar) in a side-by-side comparison chart, showing that only the new brand has the benefits to address all seven; other brands provide no more than four of the benefits. In the other spot, a woman informs her husband that they are now using the new brand of toothpaste. The husband asks whether the brand has two of the benefits presented in the previously described ad. When his wife says it does, he turns off the lights momentarily and then turns them back on and asks whether the brand has several additional benefits. This sequence is repeated until all seven benefits are presented. The audience is then told that competitors have only four out of the seven features. Thus, the two ads contain essentially the same information that is presented in either a simultaneous or sequential format. Our research would predict that if the target segment had a promotion focus, the spot using a sequential presentation would have been more effective. But because the target in the present case is more likely to have a prevention focus, the decision fit hypothesis prediction is that the spot employing the simultaneous presentation would be more persuasive. In fact, the spot featuring the simultaneous presentation was the winner in the test and was recently aired.

\section{APPENDIX}

\section{PRODUCT EVALUATION STIMULI USED IN STUDY 1}

Imagine that you were considering buying a cell phone. You are provided with product information of five different brands. The following table lists the five different brands of cell phone, one in each column, and information on five different attributes, one in each row. They sell for about the same price.

\section{Progress Decision Strategy: Lexicographic Condition}

Please examine the information for these brands of cell phone using the following strategy. Rank the importance of the four attributes. Look at the most important attribute, and choose the brand that has the best value on this attribute. If there is a tie between several brands on the most important attribute, look at the second most important attribute for the brands tied on the most important attribute. Choose the brand that has the best value on the second most important attribute. If there is still a tie between brands on the second most important attribute, look at the third most important attribute for the brands tied on the second most important attribute. Follow this procedure until you are left with only one brand. Mark it as your chosen brand. 


\section{Accuracy Decision Strategy: Equal Weight Condition}

Please examine the information for these brands of cell phone using the following strategy. Look at brand A. Compare it to the rest of the brands based on each of the attributes. Now look at brand B. Compare it to the rest of the brands based on each of the attributes. Now look at brand C. Compare it to the rest of the brands based on each of the attributes. Do so until you have looked at all the brands and at all the attributes. After you are done comparing between the brands, decide which brand you prefer most. Mark this brand as your chosen brand.

\begin{tabular}{lccccc}
\hline & \multicolumn{5}{c}{ Brand } \\
\cline { 2 - 6 } & A & B & C & D & E \\
\hline Battery talk time (hours) & 5.0 & 5.0 & 5.0 & 5.0 & 4.5 \\
Battery standby time (days) & 3.0 & 3.0 & 2.5 & 3.0 & 3.0 \\
Warranty (months) & 18 & 18 & 18 & 12 & 18 \\
Camera capability & No & Yes & Yes & Yes & Yes \\
\hline
\end{tabular}

\section{REFERENCES}

Aaker, Jennifer L. and Angela Y. Lee (2001), “'I' Seek Pleasures and 'We' Avoid Pains: The Role of Self-Regulatory Goals in Information Processing and Persuasion," Journal of Consumer Research, 28 (June), 33-49.

Avnet, Tamar and E. Tory Higgins (2003), "Locomotion, Assessment, and Regulatory Fit: Value Transfer from 'How' to 'What,"' Journal of Experimental Social Psychology, 39 (September), 525-30.

Baron, Reuben M. and David A. Kenny (1986), "The ModeratorMediator Variable Distinction in Social Psychological Research: Conceptual, Strategic, and Statistical Considerations," Journal of Personality and Social Psychology, 51 (December), 1173-82.

Bettman, James R., Mary F. Luce, and John W. Payne (1998), "Constructive Consumer Choice Processes," Journal of Consumer Research, 25 (December), 187-217.

Broder, Arndt and Stephanie Schiffer (2003), "Take the Best versus Simultaneous Feature Matching: Probabilistic Inferences from Memory and Effects of Representation Format," Journal of Experimental Psychology: General, 132, 277-93.

Carlson, Kurt A., Margaret G. Meloy, and J. Edward Russo (2006), "Leader-Driven Primacy: Using Attribute Order to Affect Consumer Choice," Journal of Consumer Research, 32 (March), 513-18.

Carver, Charles S. and Michael F. Scheier (1998), On the Self-Regulation of Behavior, New York: Cambridge University Press.

Cesario, Joseph, Heidi Grant, and E. Tory Higgins (2004), "Regulatory Fit and Persuasion: Transfer from 'Feeling Right,"' Journal of Personality and Social Psychology, 86 (3), 388-404.

Coupey, Eloise (1994), "Restructuring: Constructive Processing of Information Displays in Consumer Choice," Journal of Consumer Research, 21 (June), 83-99.

Crowe, Ellen and E. Tory Higgins (1997), "Regulatory Focus and Strategic Inclinations: Promotion and Prevention in Decision Making," Organizational Behavior and Human Decision Processes, 69 (February), 117-32.

Förster, Jens, E. Tory Higgins, and Amy T. Bianco (2003), "Speed/
Accuracy Decisions in Task Performance: Built-in Trade-Off or Separate Strategic Concerns?" Organizational Behavior and Human Decision Processes, 90 (1), 148-64.

Friedman, Ronald S. and Jens Förster (2001), "The Effects of Promotion and Prevention Cues on Creativity," Journal of Personality and Social Psychology, 81 (December), 1001-13.

Gilbert, Daniel T. and J. Gregory Hixon (1991), "The Trouble with Thinking: Activation and Application of Stereotypic Beliefs," Journal of Personality and Social Psychology, 60 (April), 509-17.

Heine, Steven J., Darrin R. Lehman, Hazel R. Markus, and Shinobu Kitayama (1999), "Is There a Universal Need for Positive Self-Regard?” Psychological Review, 106 (4), 766-94.

Higgins, E. Tory (1997), "Beyond Pleasure and Pain," American Psychologist, 52 (December), 1280-1300.

- (2000), "Making a Good Decision: Value from Fit," American Psychologist, 55 (November), 1217-30.

Higgins, E. Tory, Lorraine C. Idson, Antonio L. Freitas, Scott Spiegel, and Daniel C. Molden (2003), "Transfer of Value from Fit," Journal of Personality and Social Psychology, 84 (December), 1140-53.

Kahneman, Daniel and Shane Frederick (2002), "Representativeness Revisited: Attribute Substitution in Intuitive Judgment," in Heuristics and Biases: The Psychology of Intuitive Judgment, ed. Thomas Gilovich, Dale Griffin, and Daniel Kahneman, New York: Cambridge University Press, 49-81.

Lee, Angela Y. and Jennifer L. Aaker (2004), "Bringing the Frame into Focus: The Influence of Regulatory Fit on Processing Fluency and Persuasion," Journal of Personality and Social Psychology, 86 (February), 205-18.

Lee, Angela Y. and Aparna Labroo (2004), "Effects of Conceptual and Perceptual Fluency on Affective Judgment," Journal of Marketing Research, 41 (May), 151-65.

Liberman, Nira, Lorraine Chen Idson, Christopher J. Camacho, and E. Tory Higgins (1999), "Promotion and Prevention Choices between Stability and Change," Journal of Personality and Social Psychology, 77 (December), 1135-45.

Payne, John, James R. Bettman, and Eric J. Johnson (1988), “Adaptive Strategy Selection in Decision Making," Journal of Experimental Psychology, 14 (3), 534-52.

(1993), The Adaptive Decision Maker, New York: Cambridge University Press.

Schwarz, Norbert (2004), "Meta-cognitive Experiences in Consumer Judgments and Decision Making," Journal of Consumer Psychology, 14 (4), 332-48.

Sengupta, Jaideep and Rongrong Zhou (2007), "Understanding Impulsives' Choice Behaviors: The Motivational Influences of Regulatory Focus," Journal of Marketing Research, 44 (May), 297-308.

Sloman, Steven A. (1996), "The Empirical Case for Two Systems of Reasoning," Psychological Bulletin, 119 (1), 3-22.

Sobel, Michael E. (1982), "Asymptotic Confidence Intervals for Indirect Effects in Structural Equation Models," in Sociological Methodology, ed. Samuel Leinhardt, San Francisco: Jossey-Bass, 290-312.

Tybout, Alice M., Brian Sternthal, Prashant Malaviya, Georgios Bakamitsos, and Se-Bum Park (2005), "Information Accessibility as a Moderator of Judgments: The Role of Content versus Retrieval Ease," Journal of Consumer Research, 32 (June), 76-85.

Wänke, Michaela, Gerd Bohner, and Andreas Jurkowitsch (1997), "There Are Many Reasons to Drive a BMW: Does Imagined Ease of Argument Generation Influence Attitudes?" Journal of Consumer Research, 24 (September), 170-77. 
Copyright of Journal of Consumer Research is the property of Journal of Consumer Research. Inc. and its content may not be copied or emailed to multiple sites or posted to a listserv without the copyright holder's express written permission. However, users may print, download, or email articles for individual use. 